\title{
PREFERÊNCIA DE Atta laevigata F. SMITH, 1858 (HYMENOPTERA: FORMICIDAE) POR DIFERENTES ESPÉCIES E HÍBRIDOS DE EUCALIPTOS
}

\author{
Joilson Onofre Pereira dos Santos ${ }^{1}$, Otávio Peres Filho ${ }^{1}$, Marcelo Dias de Souza ${ }^{2}$, Alberto
} Dorval ${ }^{1}$

\footnotetext{
${ }^{1}$ Universidade Federal de Mato Grosso, e-mail: joilson.eng@gmail.com, peres@ufmt.br, adorval@terra.com.br
}

${ }^{2}$ Universidade Federal do Paraná, e-mail: marcelo.dias@florestal.eng.br

\section{RESUMO}

Este trabalho teve como objetivo avaliar a preferência de Atta laevigata por diferentes espécies, híbridos e clones de eucaliptos provenientes de diferentes localidades do estado do Mato Grosso. A atratividade foi avaliada determinando-se a quantidade de discos foliares transportados de cada tratamento. A. laevigata apresentou maior preferência ao híbrido Eucalyptus urophylla x Eucalyptus grandis (urograndis). As espécies com menores quantidades de discos foliares carregados pelas formigas foram Eucalyptus camaldulensis e Corymbia citriodora.

Palavras-chave: Eucalipto, formigas cortadeiras, forrageamento, seletividade

\section{PREFERENCE OF Atta laevigata F. SMITH, 1858 (HYMENOPTERA: FORMICIDAE) TO DIFFERENT SPECIES AND HYBRIDS OF EUCALYPTUS}

\begin{abstract}
This work aimed to evaluate the preference of Atta laevigata by different species, hybrids and eucalyptus clones from different locations of the State of Mato Grosso, Brazil. The attraction was evaluated by determining the amount of transported leaf discs per treatment. A. laevigata showed higher preference to the hybrid Eucalyptus grandis x Eucalyptus urophylla (urograndis). The species with lower amounts of leaf discs carried by ants were Eucalyptus camaldulensis and Corymbia citriodora.
\end{abstract}

Keywords: Eucalyptus, leaf-cutting ants, foraging, selectivity 


\section{INTRODUÇÃO}

Nos últimos anos, o setor florestal brasileiro apresenta grande crescimento, tanto em quantidade de plantações com fins comerciais, quanto em rentabilidade. Segundo dados da Associação Brasileira de Produtores de Florestas Plantadas, a cada ano o setor florestal demonstra crescimento, principalmente na cultura de eucalipto (ABRAF, 2013). Para Santana \& Couto (1990), em plantios de eucalipto podem ocorrer problemas que, muitas vezes, comprometem o sucesso do reflorestamento, tais como a presença de pragas na cultura, sendo que entre os diversos insetos-praga associados à cultura do eucalipto, destacamse as formigas cortadeiras devido aos constantes ataques que essas formigas causam em diversas espécies e híbridos desse vegetal.

As formigas cortadeiras do gênero Atta (saúva) e Acromyrmex (quenquém) são consideradas pragas constantes nas diversas fases do desenvolvimento da floresta. De acordo com Zanetti et al. (2002), o ataque realizado por formigas cortadeiras é de maneira intensa e constante, podendo ocorrer danos em qualquer fase do desenvolvimento do vegetal, ocasionados por cortes de folhas, brotos, ramos finos e flores, os quais são carregados para o interior de ninhos subterrâneos para alimentação de um fungo simbionte, Leucoagaricus gongylophorus (Möller) Singer, queas formigas utilizam como alimento.

A capacidade de colonização e forrageamento dessas formigas aliada, principalmente, ao ambiente que sofreu desequilíbrio ecológico provocado pelo desmatamento e implantação de monoculturas, propiciam condições ideais para o estabelecimento e a proliferação das colônias, favorecendo também o aumento da densidade populacional causando diversos prejuízos a cultura (CHERRETT, 1986; DELLA LUCIA; FOWLER, 1993).

Anjos et al. (1998) relataram que o desfolhamento provocado por formigas cortadeiras causam prejuízos de $13 \%$ da colheita e em ecossistemas tropicais, as formigas cortadeiras consomem cerca de $15 \%$ da produção florestal.

A capacidade das diferentes espécies vegetais usadas para o desenvolvimento do fungo simbionte dessas formigas depende da sua composição química, que é diferente entre as espécies de plantas, sendo estas, influenciada pelas condições culturais, fatores climáticos e estágiode desenvolvimento da planta (CASTELLANI et al., 2007). Essas diferenças afetam as preferências das formigas e o 
desenvolvimento do fungo, além de ser extremamente difíceis de serem medidas com precisão, mas são detectáveis pelo efeito na taxa de crescimento do fungo (MUDD \& BATEMAN, 1979).

Peres Filho et al. (2002), estudando a preferência de carregamento de 42 espécies vegetais por Atta sexdens rubropilosa Forel, constataram que Eucalyptus grandis W. Hill ex-Maiden teve menos disco foliares carregados em comparação com as demais espécies florestais nativas e exóticas, em condições de laboratório. Segundo os autores, dependendo da diversidade de espécies florestais testadas, o comportamento das formigas pode ser alterado. Os autores ainda destacam que os compostos presentes nas folhas das diferentes espécies podem interferir na preferência de ataque pelas formigas cortadeiras.

Dessa forma, o estudo teve como objetivo avaliar a preferência de Atta laevigata (F. Smith) por diferentes espécies, híbridos e clones de eucaliptos provenientes de diferentes localidades do estado do Mato Grosso, em condições de campo, a fim de fornecer informações importantes sobre a influência das localidades e dos diferentes tratamentos em relação à preferência do carregamento foliar para o interior do formigueiro..

\section{MATERIAL E MÉTODOS}

Para os testes de atratividade foram coletadas duas espécies, 17 clones e dois híbridos de Eucalyptus provenientes de três localidades distintas do estado de Mato Grosso (Tabela 1). Todos os indivíduos amostrados foram plantados no período de Novembro-2008 a Março-2009, de acordo com projeto experimental da Associação dos Reflorestadores do Estado do Mato Grosso (Arefloresta).

As árvores foram plantadas nos municípios de Dom Aquino, Chapada dos Guimarães, Sinop, Itiquira, Nobres, Cáceres e Santa Rita do Trivelato. Para efeito de representatividade, foram escolhidos três locais para coleta do material e aplicação dos testes, sendo selecionados para coleta os plantios situados nos municípios de Itiquira, Santa Rita do Trivelato e Sinop, por serem os locais mais ao Sul $\left(17^{\circ} 17^{\prime} 34,4^{\prime \prime S} / 55^{\circ}\right.$ $\left.10^{\prime} 22,8^{\prime \prime} \mathrm{W}\right)$, Centro (14\% $02^{\prime} 05,5^{\prime} \mathrm{S} / 55^{\circ}$ 03' 30,8” W) e Norte ( $11^{\circ} 51^{\prime} 59,9$ ” S / 55 27 '58,7” W), respectivamente, do estado de Mato Grosso. 


\section{PREFERÊNCIA DE Atta laevigata F. SMITH, 1858 (HYMENOPTERA: FORMICIDAE) POR \\ DIFERENTES ESPÉCIES E HÍBRIDOS DE EUCALIPTO}

Tabela 1. Espécies, híbridos e clones de eucalipto utilizadas nos testes de preferência de Atta laevigata ao carregamento foliar de diferentes regiões no Estado de Mato Grosso, 2011.

\begin{tabular}{lc}
\hline Espécie/Híbrido & Tipo de plantio \\
\hline Eucalyptus urophyllax Eucalyptus grandis (EUCA 103) & Clonal \\
Eucalyptus urophylla x Eucalyptus grandis(EUCA 105) & Clonal \\
Eucalyptus grandis x Eucalyptus resinifera (EUCA 608) & Clonal \\
Eucalyptus camaldulensis (S23) & Clonal \\
Eucalyptus urophylla x Eucalyptus grandis(H13) & Clonal \\
Eucalyptus camaldulensis x Eucalyptus spp. (1277) & Clonal \\
Eucalyptus urophylla x Eucalyptus grandis (Urograndis Acesita) & Seminal \\
Eucalyptus urophylla x Eucalyptus grandis (GG100) & Clonal \\
Eucalyptus urophylla x Eucalyptus grandis (IO42) & Clonal \\
Eucalyptus urophylla x Eucalyptus grandis (Urograndis Luiz A.) & Seminal \\
Corymbia citriodora (Citriodora) & Seminal \\
Eucalyptus camaldulensis (Camaldulensis) & Seminal \\
Eucalyptus urophylla x Eucalyptus grandis (F1H13) & Clonal \\
Eucalyptus camaldulensis (F3C219) & Clonal \\
Eucalyptus urophylla x Eucalyptus grandis (F8 Copener) & Clonal \\
Eucalyptus urophyllax Eucalyptus grandis (F11 Copener) & Clonal \\
Eucalyptus urophyllax Eucalyptus grandis (H77) & Clonal \\
Eucalyptus urophyllax Eucalyptus grandis (I144) & Clonal \\
Eucalyptus urophyllax Eucalyptus grandis (I224) & Clonal \\
Urograndis x E. camaldulensis (VM 01) & Clonal \\
Corymbia citriodora (LCA019) & Seminal \\
\hline
\end{tabular}

No solo do plantio situado no município de Itiquira, os percentuais de areia, silte e argila são de $87,7 \%, 3,2 \%$ e 9,1\% respectivamente, sendo caracterizado como de textura areia franca. A região possui precipitação média anual de 1.500 mm, com intensidade máxima de dezembro a fevereiro. Temperatura média anual de $22^{\circ} \mathrm{C}$, maior máxima $40^{\circ} \mathrm{C}$ e menor mínima $0^{\circ} \mathrm{C}$. No solo do plantio situado no município de Santa Rita do Trivelato os percentuais de areia, silte e argila são $86,1 \%$, $4,8 \%$ e $9,1 \%$ respectivamente, sendo caracterizado como de textura areia franca. A região possui precipitação média anual de $2.000 \mathrm{~mm}$, com intensidade máxima em janeiro, fevereiro e março. Temperatura média anual de $24^{\circ} \mathrm{C}$, maior máxima $38^{\circ} \mathrm{C}$ e menor $4^{\circ}$ C.O solo do plantio situado no município de Sinop possui percentuais de areia, silte e argila em 47,6\%, 10,1\% e $42,3 \%$ respectivamente, sendo caracterizado 
como de textura franco argilosa. A região possui precipitação média de $2.500 \mathrm{~mm}$, com maior intensidade nos meses de janeiro, fevereiro e março. Temperatura média anual de $24^{\circ} \mathrm{C}$, maior máxima $40^{\circ} \mathrm{C}$ e menor $0^{\circ} \mathrm{C}$ (RESENDE et al., 2002; MARQUES, 2012, ROSA et al., 2007; SANTA RITA DO TRIVELATO, 2008)

Nos locais de plantio foram implantados, de acordo com o projeto experimental da Arefloresta, quatro repetições de 49 árvores para cada tratamento, sendo que foi coletado o material foliar do terço inferior da árvore central do primeiro bloco de cada tratamento. No caso de falhas, foi feito o caminhamento em sentido horário até que se encontrasse um exemplar vivo, segundo metodologia aplicada por Stradling (1978) (Figura 1-A). As folhas coletadas foram mantidas em sacolas de polietileno e armazenadas em caixas térmicas contendo gelo para evitar alterações no conteúdo de umidade foliar. Além disso, entre o gelo e as sacolas com as folhas foi colocada uma pequena camada de folhas para evitar o contato direto do gelo com as folhas dos tratamentos.

$\mathrm{O}$ experimento foi realizado em Outubro de 2011 em condições de campo, da qual o teste de atratividade com formigueiro ativo de $A$. laevigata (saúva cabeça-de- vidro), com dimensões de 4,9 $\mathrm{m} \mathrm{x} \mathrm{7,7} \mathrm{m}$ $\left(37,73 \mathrm{~m}^{2}\right)$ situado em um fragmento de cerrado localizado no entorno do Parque Nacional da Chapada dos Guimarães. As coordenadas do formigueiro utilizado são $15^{\circ} 21^{\prime} 19,52 " \mathrm{~S} 55^{\circ} 55^{\prime} 46,91^{\prime \prime} \mathrm{W}$.

Os testes de atratividade foram realizados entre às 18:00 $\mathrm{h}$ e 22:00 $\mathrm{h}$, ou seja, sempre no período noturno, uma vez que, de acordo com observações de campo, é o período em que as formigas saem para cortar as folhas de espécies vegetais. Foi utilizada a metodologia de Cherret \& Seaforth (1970) utilizando-se placas de alumínio de $15 \mathrm{~cm}$ x $15 \mathrm{~cm}$ com 100 quadrículas de $1,0 \mathrm{~cm} \times 1,0 \mathrm{~cm}$ e bordaduras de $2,5 \mathrm{~cm}$ (Figura 1-B). As placas foram devidamente lavadas com sabão neutro e esterilizadas em estufa a $150{ }^{\circ} \mathrm{C}$, pelo período de uma hora, antes e após qualquer utilização.

As placas foram numeradas de 1 a 21, dos quais foram colocados 25 discos foliares de cada tratamento, sendo que em que cada número de placa correspondia a um determinado tratamento. As amostras foliares foram obtidas de folhas do terço inferior da copa das árvores, sendo feitos disco foliares com um vazador metálico de forma circular, com diâmetro de $0,5 \mathrm{~cm}$. 

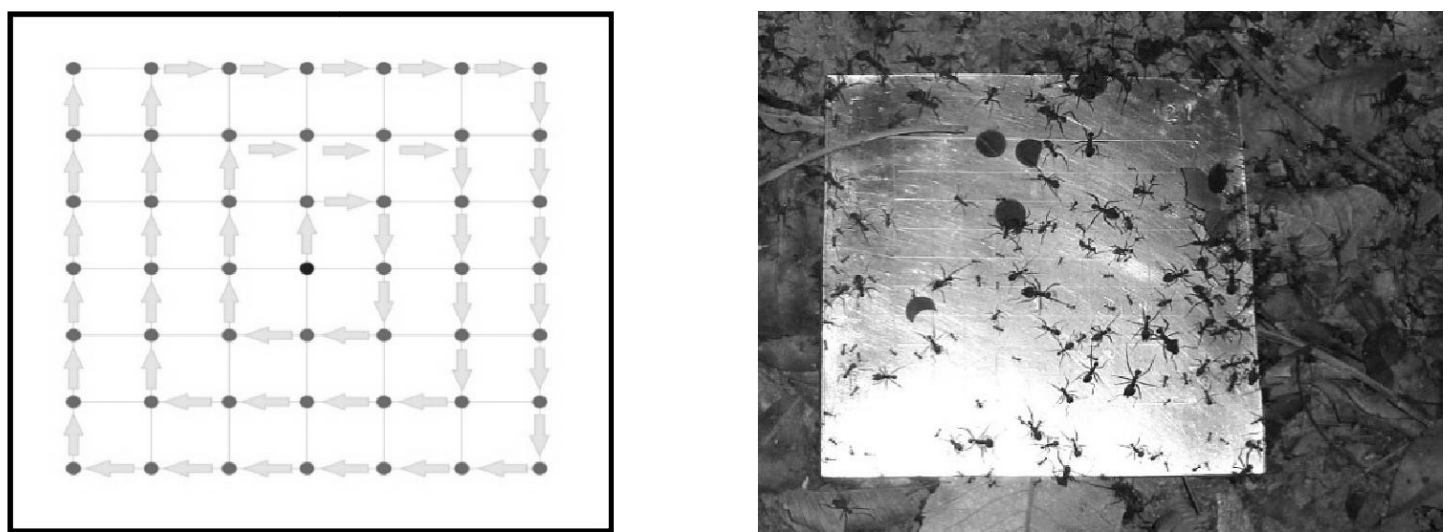

Figura 1. (A) Diagrama de coleta foliar em cada talhão dos tratamentos (Stradling,1978). (B) Placas de alumínio contendo 100 quadrículas para os discos foliares (FENF/UFMT, 2011).

As placas foram dispostas a cinco centímetros de distância da trilha ativa do formigueiro de forma aleatória, sendo disponibilizados às saúvas por um período de 20 minutos ou até que a última amostra de qualquer um dos tratamentos tivesse sido carregada. Foram realizadas quatro repetições por tratamento, sendo avaliado por quatro dias. Para as análises, as repetições foram consideradas a média dos quatro dias para cada repetição.

$\mathrm{O}$ delineamento foi inteiramente casualizado em esquema fatorial (Eucaliptos $\mathrm{X}$ Região), com quatro repetições, das quais os dados foram submetidos à análise de variância (ANOVA) em que as medias dos tratamentos foram comparadas pelo teste de Scott-Knottao nível de 5\% de probabilidade de erro. A variável avaliada foi correspondente o percentual de discos foliares retirados das placas e transportados para o interior do formigueiro, das quais passaram pelo teste de normalidade (Kolmogorov D: Normal test) e homogeneidade de variância (Bartlett) e transformados pela fórmula , conforme preconizado por Banzatto \& Kronka (2006). As análises estatísticas foram efetuadas utilizando-se o software Sisvar (FERREIRA, 2008).

\section{RESULTADOS E DISCUSSÃO}

Foi possível constatar que as formigas apresentaram preferência diferenciada em relação aos eucaliptos provenientes das regiões, pois os tratamentos com maiores percentuais de carregamento foram diferentes para cada região. $\mathrm{O}$ tratamento com maior percentual de carregamento foi o clone I144 da região de Itiquira, com media de $95 \%$ de carregamento (Tabela 2). O tratamento com menor 
percentual de carregamento da região de Itiquira foi proveniente dos plantios dos clones F1H13 com media de 4\%.

É comum as formigas apresentarem preferência diferenciada entre os eucaliptos pois, de acordo com Della Lucia et al. (1995), espécies de Eucalyptus spp. contêm compostos secundários, em diferentes teores, e apresentam características físicas diversas em suas folhas. Portanto, é de se esperar diferenças na preferência de corte pelas formigas cortadeiras.

Os plantios situados na região de Santa Rita do Trivelato tiveram problemas com condições edafoclimáticas, devido ao longo período de estiagem no ano de 2009 que resultou na morte de alguns blocos dos tratamentos EUCA 608, H77 e I224, sendo que o tratamento com maior percentual de carregamento foi o F8 Copener. Já na região de Sinop houve maior preferência das formigas pelos clones $\mathrm{H} 13$ e ainda todas as médias de carregamento pelas formigas acima de $41 \%$, são clones do híbrido urograndis (Eucalyptus urophylla $\mathrm{x}$ Eucalyptus grandis).

Nas três regiões avaliadas é possível observar que os tratamentos contendo material de Eucalyptus urophylla S.T. Blake ou E. grandis apresentaram as maiores médias de percentual carregamento pelas formigas (Figura 2).

Santana \& Couto (1990), ao avaliar a preferência de $A$. laevigata por diversos clones de eucalipto, verificaram que, entre os clones procedentes de E. grandis, houve variações de 57\% na preferência de carregamento foliar. De acordo Viana et al. (2010), Eucalyptus grandis possui diferentes teores de compostos químicos na cera epicuticular nas folhas, afetando na preferência de corte pelas formigas.

Uma hipótese que pode explicar a baixa preferência ao corte por algumas espécies de eucalipto pode estar relacionada com os compostos secundários com ação repelente presente nos óleos essenciais de algumas procedências de eucaliptos. Os óleos essenciais de eucalipto são ricos em terpenoides que podem atuar como repelente às formigas cortadeiras, pois quando expostas ao óleo essencial, apresentam alterações de comportamento (MARSARO JÚNIOR et al., 2004).

Como houve mortalidade de tratamentos nos plantios de Santa Rita do Trivelato, onde a análise fatorial foi de $18 \times 3$ (Eucaliptos x regiões), foi possível constatar diferenças significativas entre os tratamentos e as regiões. 
Tabela 2. Percentual médio de discos foliaresdos diferentes materiais de Eucaliptos carregados por Atta laevigata provenientes das diferentes regiões no Estado de Mato Grosso, 2011.

\begin{tabular}{|c|c|c|c|}
\hline \multirow{2}{*}{ Tratamentos } & \multicolumn{3}{|c|}{ Regiões } \\
\hline & Itiquira & Trivelato & Sinop \\
\hline EUCA 103 & 58 & 13 & 9 \\
\hline EUCA 105 & 89 & 86 & 73 \\
\hline EUCA 608 & 14 & - & 22 \\
\hline $\mathrm{S} 23$ & 50 & 23 & 8 \\
\hline H13 & 89 & 67 & 87 \\
\hline 1277 & 32 & 36 & 67 \\
\hline Urograndis (Acesita) & 45 & 17 & 17 \\
\hline GG100 & 38 & 88 & 74 \\
\hline $\mathrm{IO} 42$ & 79 & 19 & 17 \\
\hline Urograndis (Luiz A.) & 65 & 41 & 19 \\
\hline Citriodora & 18 & 3 & 1 \\
\hline Camaudulensis & 12 & 7 & 3 \\
\hline F1H13 & 4 & 10 & 13 \\
\hline F3C219 & 82 & 10 & 12 \\
\hline F8 Copener & 60 & 90 & 31 \\
\hline F11 Copener & 5 & 32 & 19 \\
\hline $\mathrm{H} 77$ & 32 & - & 59 \\
\hline $\mathrm{I} 144$ & 95 & 13 & 12 \\
\hline $\mathrm{I} 224$ & 86 & - & 68 \\
\hline VM 01 & 15 & 32 & 22 \\
\hline Cit / LCA019 & 20 & 30 & 4 \\
\hline
\end{tabular}




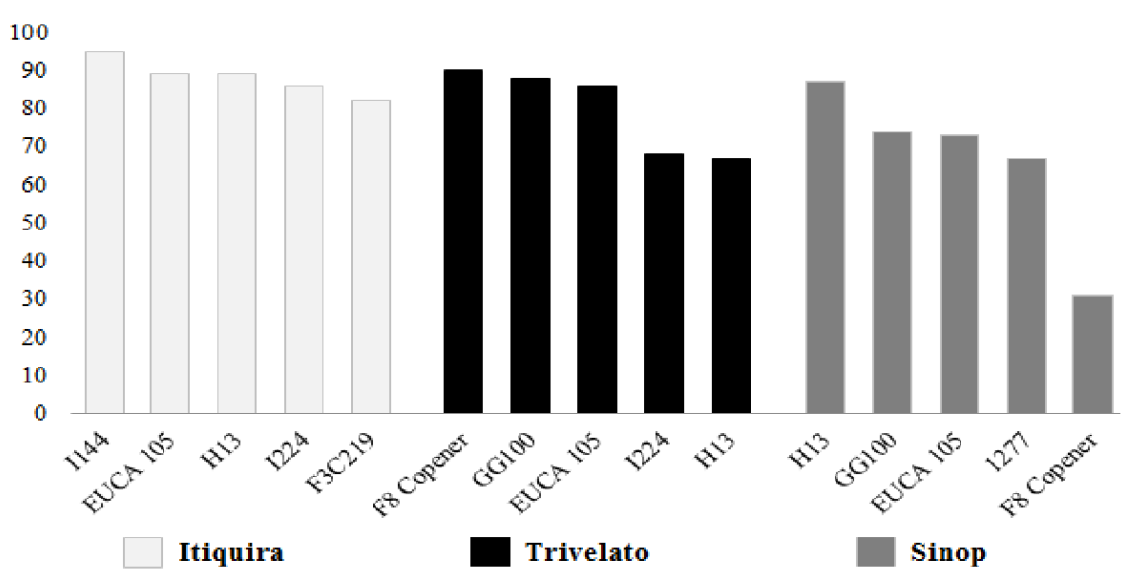

Figura 2. Gráfico do percentual médio de discos foliares carregados por Atta laevigata de diferentes materiais de eucaliptos dos municípios de Itiquira, Santa Rita do Trivelato e Sinop, 2011.

Os plantios com maiores médias de carregamento foram os situados em Itiquira diferindo significativamente das demais regiões (Tabela 3).

As formigas apresentaram preferência diferenciada entre os tratamentos de acordo com o local de plantio, como os clones EUCA 105 foi o tratamento que apresentou as maiores médias percentuais de carregamento da qual se destacam os plantios da região de Itiquira e Sinop diferindo apenas de Santa Rita do Trivelato. Outro fator observado na analise conjunta, é que os tratamentos com menor preferência são provenientes de plantios seminais, Eucalyptus camaldulensis e Corymbia citriodora, principalmente dos plantios situados em Santa Rita do Trivelato e Sinop.

$$
\text { Rezende \& Della-Lucia (1999) }
$$
afirmaram que folhas contendo alto teor de óleos essenciais e ainda mudas apresentando alta pilosidade nas folhas, podem servir como uma barreira ao corte e carregamento pelas formigas cortadeiras, sendo essa uma das caracteristicas de C. citriodora.

Chrispim \& Ramos (2007) afirmaram que a não preferência dos vegetais pelos insetos pode estar relacionada aos fatores climáticos e edáficos que afetam o desenvolvimento do vegetal, tais como umidade, temperatura e disponibilidade de nutrientes, sais minerais e até mesmo plantas adjacentes presentes no plantio. Os autores ainda enfatizam que esses fatores podem contribuir para a manifestação de resistência nos vegetais, estabelecendo um microclima favorável ou desfavorável para o inseto em que pode atenuar ou inibir o ataque respectivamente. 


\section{PREFERÊNCIA DE Atta laevigata F. SMITH, 1858 (HYMENOPTERA: FORMICIDAE) POR DIFERENTES ESPÉCIES E HÍBRIDOS DE EUCALIPTO}

Tabela 3. Análise fatorial do percentual médio de discos foliares dos diferentes materiais de Eucaliptos provenientes de Santa Rita do Tivelato, Itiquira e Sinop, carregados pelas formigas, 2011.

\begin{tabular}{|c|c|c|c|}
\hline \multirow{2}{*}{ Eucaliptos } & \multicolumn{3}{|c|}{ Região $^{1}$} \\
\hline & Itiquira & Trivelato & Sinop \\
\hline EUCA 103 & $49,62 \pm 3,01 \mathrm{cA}$ & $15,06 \pm 3,05 \mathrm{eB}$ & $20,88 \pm 3,89 \mathrm{~dB}$ \\
\hline EUCA 105 & $73,35 \pm 10,46$ aA & $58,82 \pm 2,55$ b B & $68,28 \pm 6,81$ aA \\
\hline $\mathrm{S} 23$ & $45,01 \pm 4,19 \mathrm{cA}$ & $16,16 \pm 6,81$ eC & $28,60 \pm 5,17 \mathrm{cB}$ \\
\hline H13 & $73,22 \pm 11,88$ aA & $69,24 \pm 2,74$ aA & $55,88 \pm 4,28$ b B \\
\hline 1277 & $34,32 \pm 4,38 \mathrm{~dB}$ & $54,98 \pm 5,55$ bA & $36,84 \pm 2,35 \mathrm{cB}$ \\
\hline Urograndis (Acesita) & $42,11 \pm 4,35 \mathrm{dA}$ & $24,32 \pm 2,76 \mathrm{~dB}$ & $24,18 \pm 6,31 \mathrm{~dB}$ \\
\hline GG100 & $38,02 \pm 6,12 \mathrm{dC}$ & $59,38 \pm 1,20$ bB & $70,47 \pm 7,75$ aA \\
\hline $\mathrm{IO} 42$ & $62,79 \pm 3,57 \mathrm{bA}$ & $23,97 \pm 2,76 \mathrm{~dB}$ & $25,76 \pm 3,83 \mathrm{~dB}$ \\
\hline Urograndis (Luiz A.) & $53,73 \pm 2,58 \mathrm{cA}$ & $25,76 \pm 5,92 \mathrm{dC}$ & $39,71 \pm 2,01 \mathrm{cB}$ \\
\hline Citriodora & $24,93 \pm 11,57$ eA & $2,88 \pm 3,89 \mathrm{fB}$ & $8,65 \pm 2,44$ eB \\
\hline Camaudulensis & $19,74 \pm 5,13$ eA & $6,99 \pm 5,76 \mathrm{fB}$ & $14,94 \pm 4,78$ eA \\
\hline F1H13 & $7,95 \pm 11,83 \mathrm{fB}$ & $20,96 \pm 5,76 \mathrm{dA}$ & $18,34 \pm 6,49 \mathrm{dA}$ \\
\hline F3C219 & $65,06 \pm 5,24 \mathrm{bA}$ & $19,52 \pm 5,68 \mathrm{~dB}$ & $17,95 \pm 4,99 \mathrm{~dB}$ \\
\hline F8 Copener & $50,81 \pm 3,04 \mathrm{cB}$ & $33,80 \pm 8,31 \mathrm{cC}$ & $72,25 \pm 3,61$ aA \\
\hline F11 Copener & $11,09 \pm 2,76 \mathrm{fC}$ & $25,68 \pm 4,23 \mathrm{~dB}$ & $34,42 \pm 2,01 \mathrm{cA}$ \\
\hline I144 & $77,23 \pm 3,45$ aA & $19,92 \pm 9,84 \mathrm{~dB}$ & $20,48 \pm 5,13 \mathrm{~dB}$ \\
\hline VM 01 & $22,45 \pm 1,49$ eB & $27,85 \pm 3,40 \mathrm{cB}$ & $34,42 \pm 6,97 \mathrm{cA}$ \\
\hline Cit / LCA019 & $26,28 \pm 3,76$ eA & $9,87 \pm 2,21 \quad \mathrm{fB}$ & $33,15 \pm 3,24 \mathrm{cA}$ \\
\hline Total & $43,21 \pm 8,73 \quad \mathrm{~A}$ & $28,62 \pm 4,04 \mathrm{C}$ & $37,73 \pm 4,21 \quad \mathrm{~B}$ \\
\hline $\mathrm{F}_{\text {Região }}$ & & $126,82 * *$ & \\
\hline $\mathrm{F}_{\text {Interação }}$ & & $27,91 * *$ & \\
\hline $\mathrm{CV}(\%)$ & & 15,54 & \\
\hline
\end{tabular}

${ }^{1}$ Dados transformados em: $\operatorname{arcsen} \sqrt{(\mathbf{X} \backslash \mathbf{1 0 0})}$

${ }^{* *}$ Significativo a $1 \%$ de probabilidade de erro

Médias seguidas pela mesma letra minúsculas nas colunas e maiúsculas nas linhas não diferem estatisticamente entre si, ao nível de 5\% de probabilidade de erro pelo teste de Scott-Knott.

Nos plantios situados em Itiquira, apesar de não ter ocasionado a morte geral de tratamentos, como em Santa Rita do Trivelato, ocorreu mortalidade nos talhões dos tratamentos, entretanto esta mortalidade ocorreu em detrimento ao ataque de
Glycaspis brimblecombei Moore (psilídeode-concha) que é constante na região e ainda associado à estiagem que ocorre no município. Este fator pode contribuir para aumentar a preferência de corte pelas formigas dos plantios dessa região, pois de 
acordo com Dahlsten (1999), a infestação do psilídeo-de-concha nas árvores acarreta estresse do vegetal, o que as tornam susceptíveis ao ataque de outras pragas, além de ocorrência de doenças.

\section{CONCLUSÕES}

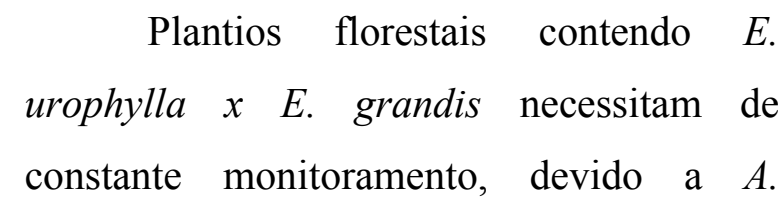
laevigata apresentar preferência de corte por esses plantios. As condições ambientais dos plantios situados na região de Itiquira podem contribuir para o desenvolvimento de eucaliptos susceptíveis ao ataque de formigas cortadeiras. Plantio com espécies seminais puros, como E. calmaldulensis e $C$. citriodora apresentam resistência ao ataque de A. laevigata, em comparação com plantios clonais.

\section{REFERÊNCIAS BIBLIOGRÁFICAS}

ANJOS, N.; DELlA LUCIA, T. M. C.; MAYHÉ NUNES, A. J. 1998. Guia prático sobre formigas cortadeiras em reflorestamento. Ponte Nova, MG: Graff Cor. 100p.

ASSOCIAÇÃO BRASILEIRA DE PRODUTORES DE FLORESTAS PLANTADAS (ABRAF). 2013. Anuário estatístico da ABRAF : ano base 2012. Brasília, 142p.

BANZATTO, D. A.; KRONKA, S. N. 2006.Experimentação Agrícola. $4^{\mathrm{a}} \mathrm{Ed}$. Jaboticabal, SP. Funep, 237p.

CASTELlANI, M. A.; FORTI, L. C.; FENILLE, R. C.; MOREIRA, A.A.;
ANDRADE, A. P. P.; NOVCAES, Q. S. 2007. Isolation and growth of the symbiotic fungus of Atta capiguara (Hymenoptera:

Formicidae). Sociobiology, Feira de Santana. v.50, n.3, p.959-972.

CHERRET, J. M. 1986. History of the leafcutting ant problems. In: LOFGREN, C.S.; VANDER MEER, R.K. (Eds.) Fire ants and leaf cutting ants: biology and management. Boulder, Westview Press: p.10-17.

CHERRET, J.M.; SEAFORTH, C.E. 1970. Phytochemical arrestants for leaf-cutting ants, Atta cephalotes (L.) and Acromyrmex octospinosus (Reich), with some notes on the ants responses. Bulletin of Entomological Research.Cambridge,v. 59, p. 615-625.

CHRISPIM, T. P.; RAMOS, J. M. 2007. Revisão de literatura: resistência de plantas a insetos. Revista científica eletrônica de engenharia florestal (online). v.10, 10p.

DAHLSTEN, D.L. 1999. Biological control of the red gum lerp psyllid, a pest of Eucalyptus species in California. The regents of the University of California.

DELLA LUCIA, T. M. C.; FOWLER, H. G. 1993. As formigas cortadeiras. In: DELlA LUCIA, T. M. C. (Ed.). As formigas cortadeiras. Viçosa: Editora Folha de Viçosa, p.01-03. 262p.

DELLA LUCIA, T. M. C.; OLIVEIRA, M. A.; ARAÚJO, M. S.; VILELA. E. F. 1995. Avaliação da não-preferência da formiga cortadeira Acromyrmex subterraneus Forel ao corte de Eucalyptus. Revista árvore. Viçosa, v.19, n.1, p.92-99.

FERREIRA, D. F. 2008. SISVAR: um programa para análises e ensino de estatística. Revista Symposium, Lavras, v.6, p.36-41.

MARSARO JÚNIOR, A. L.; SOUZA, R. C.; DELLA LUCIA, T. M. C.; FERNANDES, J. B.; SILVA, M.F.G.F.; 
VIEIRA, P. C. 2004. Behavioral changes in workers of the leaf-cutting ant Atta sexdens rubropilosa induced by chemical components of Eucalyptus maculata leaves. Journal of chemical ecology, Heidelberg, v.30, n.9, p.1771-1780.

MARSARO JÚNIOR, A. L.; RUGAMA, A. J. M.; LIMA, C. A.; DELLA LUCIA T. M. C. 2007. Preferência de corte de Eucalyptus spp. por Acromyrmex laticeps nigrosetosus Forel 1908 (Hymenoptera: Formicidae) em condições de laboratório. Ciência Florestal, Santa Maria, v. 17, n. 2, p. 171-174.

MUDD, A.; BATEMAN, G. L. 1979.Rates of growth of the food fungus of the leafcutting ant Atta cephalotes (L.)(Hymenoptera: Formicidae) on different substrates gathered by the ants. Bulletin of Entomological Research, v.69, p.141-148.

PERES FILHO, O.; DORVAL, A.; BERTI FILHO, E.; 2002. Preferência de saúva limão, Atta sexdens rubropilosa Forel, 1908 (Hymenoptera, formicidae) a diferentes espécies florestais, em condições de laboratório. Ciência Florestal, Santa Maria, v. 12, n. 2, p. 1-7. RESENDE, M.; CURI, N.; REZENDE, S. B.; CORREAA, G. F.; 2002. Pedologia: Base para distinção de ambientes. 4 ed. - Viçosa: NEPUT, 338p.

REZENDE, M. A. A.; DELLA LUCIA, T.M.C. 1999. Is Eucalyptus citriodora attacked by leaf-cutting ants? Revista árvore. Viçosa, v.23, n.1, p.69-74.

ROSA, D. B.; DE SOUZA, R. R.; NASCIMENTO, L. A.; TOLEDO, L. G.; TOPANOTTI, D. Q.; $\quad$ DO NASCIMENTO, J. A.; 2007. A distribuição espacial das chuvas na porção centro oeste do estado de Mato Grosso - Brasil. Revista eletrônica da associação dos geógrafos brasileiros. Três Lagoas, v1, n5, p127-152.
SANTA RITA DO TRIVELATO. 2008. Mato Grosso e seus municípios, Disponível em $<$ http://www.mteseusmun icipios.com.br/NG/conteudo.php?sid=220 \&cid $=998>$. Acesso em: 30/01/2015.

SANTANA, D. L. Q.; COUTO, L. 1990. Resistência intra-específica de eucaliptos a formigas-cortadeiras. Boletim de pesquisa florestal.Colombo, n.20, p.1321.

STRADLING, D.J. 1978. The influence of size on foraging in the ant, Atta cephalotes, and the effects of some plant defense mechanism. Journal of Animal Ecology, London, 47:173-88.

VIANA, R. G.; TUFFI SANTOS, L. D.; DEMUNER, A. J.; FERREIRA, F. A.; FERREIRA,L. R.; FERREIRA, E. A.; MACHADO, A. F. L.; SANTOS, M. V. 2010. Quantificação e composição química de cera epicuticular de folhas de eucalipto. Planta Daninha. Viçosa, v. 28, n. 4, p. 753-758.

ZANETTI, R.; CARVALHO, G. A.; SANTOS, A.; SOUZA-SILVA, A.; GODOY, M. S. 2002. Manejo integrado de formigas cortadeiras. Lavras: UFLA, $16 \mathrm{p}$.

Recebido em:03\03\2015 Aceito para publicação em: 05\05\2015 